\section{Preimplantation diagnosis with the polymerase chain reaction}

\section{Scientifically possible, not widely available}

DNA amplification by the polymerase chain reaction is one of those brilliant ideas that in retrospect seem obvious. ${ }^{1}$ It depends on a well known peculiarity of DNA replication: if the two strands of DNA are designated $(+)$ and $(-)$ strands to synthesise a $(+)$ strand on a $(-)$ strand template you need not only the polymerase enzyme and the DNA monomers but also a short primer segment of the $(+)$ strand. DNA synthesis works by extending the primer. By supplying only one specific primer in vitro DNA synthesis can be made to begin always at a certain sequence. If $(+)$ and $(-)$ strand primers are supplied that recognise sequences a short distance apart on the template and are arranged so that synthesis runs from each primer towards the other the sequence between the two primers can be selectively copied. The copies may themselves serve as templates for further copying - so that in theory the amount of target DNA doubles with each round of copying.

In the laboratory this is achieved by mixing the DNA template, primer sequences, DNA monomers and polymerase and controlling the reaction by changing the temperature. At $95^{\circ} \mathrm{C}$ the two strands of DNA separate; at $60^{\circ} \mathrm{C}$ the primers anneal to their complementary sequences; and at $70^{\circ} \mathrm{C}$ the special high temperature polymerase extends the primers. Thirty cycles take two to three hours to complete and give a millionfold amplification.

The technique is transforming molecular genetics. It is simple, fast, highly specific, and unbelievably sensitive. Most of the tests at present done with Southern blotting may be done much more simply with the polymerase chain reaction. A radioactive probe is no longer needed to locate the DNA fragment of interest: after amplification it is abundant enough to be seen as a fluorescent band in an electrophoretic gel. Already the polymerase chain reaction is being applied routinely to the prenatal diagnosis of cystic fibrosis and other single gene disorders using material obtained by chorionic villus biopsy at eight weeks' gestation. ${ }^{23}$ Several groups have shown that the starting material for the polymerase chain reaction may be as little as one molecule. ${ }^{1+}$ Such sensitivity makes possible many new ventures: the genotype of individual subjects may be determined from a single hair or a dried bloodspot; extremely rare RNA transcripts or a single virus may be detected within cells; and single cells may have their sex determined ${ }^{5}$ or-as described by the group at $\mathrm{St}$ Mary's on p 22 - may be examined for genetic abnormalities. If the single cell is derived from a preimplantation embryo the method can be used for uniquely early prenatal diagnosis. How useful will this be?

Couples would have a choice between natural conception followed by chorionic villus biopsy and in vitro fertilisation with preimplantation diagnosis, using the polymerase chain reaction in each case. Couples opting for chorionic villus biopsy would have to be prepared for a termination of pregnancy in the first trimester; in those opting for in vitro fertilisation the woman would have to undergo follicle stimulation, monitoring of the ovarian cycle, egg retrieval, and embryo replacement, with a considerably less than even chance of success. ${ }^{6}$ The option of in vitro fertilisation would circumvent some, but not all, of the qualms of those who find abortion a difficult choice. Couples will need help to make these choices, and this is best done within an integrated clinical, laboratory, and counselling service.
With expert support many couples may decide to take the obstetrically less complex path of chorionic villus biopsy. This might be a relief to hard pressed teams offering in vitro fertilisation. At least 1000 pregnancies annually in Britain carry a one in four risk of producing a child affected with cystic fibrosis, and if population screening to identify them becomes feasible demand for in vitro fertilisation could overwhelm the existing services. In 1987, 5592 embryo transfers were performed in Britain; most were transferred in private facilities, ${ }^{6}$ and waiting lists for NHS treatment extend for many years.

Until the technology of in vitro fertilisation becomes much simpler adding this procedure to existing prenatal diagnosis programmes seems daunting. Coutelle et al mention, however, women who have been sterilised after the birth of an abnormal child, who subsequently wish to have more children (p 22). For these women, who would already be enrolled into in vitro fertilisation programmes, adding the polymerase chain reaction as a diagnostic step would be simple and beneficial.

ANDREW P READ Senior Lecturer

DIAN DONNAI

Department of Medical Genetics,

Consultant Geneticist

St Mary's Hospital,

Manchester M13 0JH

I Saiki RK, Gelfand DH, Stoffel S, et al. Primer-directed enzymatic amplification of DNA with a thermostable DNA polymerase. Science 1988:239:487-91.

Kogan SC, Doherty M, Gitschier J. An improved method for prenatal diagnosis of genetic diseases by analysis of amplified DNA sequences. N Engl F Med 1987;317:985-90.

Williams C, Williamson R, Coutelle C, Loeffler F, Smith J, Ivinson A. Same-day, first trimester antenatal diagnosis for cystic fibrosis by gene amplification. Lancet 1988;ii:102-3.

Li H, Gyllensten UB, Cui X, Saiki RK, Ehrlich HA, Arnheim A. Amplification and analysis of DNA sequences in single human sperm and diploid cells. Nature 1988;335:414-7.

Handyside AH, Pattinson JK, Penketh RJA, Delhanty JDA, Winston RML, Tuddenham EGD. Biopsy of human preimplantation embryos and sexing by DNA amplification. Lancet 1989;ii: $347-9$

Voluntary Licensing Authority for Human In-vitro Fertilisation and Embryology, Fourth Report. London: Voluntary Licensing Authority for Human In-vitro Fertilisation and Embryology, 1989.

\section{Psychiatric aspects of Parkinson's disease}

\section{Dementia, depression, and rarely psychosis}

Parkinson's disease commonly has psychiatric complications, which may be disabling. Cognitive impairment occurs in two forms: dementia, which affects about a tenth of patients, ${ }^{12}$ and frontal-subcortical dysfunction (a more discrete deficit of the frontal lobes), which may affect up to two thirds. ${ }^{1}$

Patients with Parkinson's disease have a higher risk of developing dementia than an age matched control group, ${ }^{2}$ but the risk applies mainly to those who develop the disease after the age of $60 .{ }^{23}$ The basis of dementia in Parkinson's disease is controversial. A combination of pathological change in both substantia nigra and the cortex may lower the threshold for developing dementia. ${ }^{+}$At the extremes Alzheimer's disease may complicate Parkinson's disease, ${ }^{5}$ and parkinsonism occurs in about two thirds of patients with Alzheimer's disease. ${ }^{6}$ Some recent evidence suggests, however, that the characteristic Lewy body disease of Parkinson's disease may be more diffuse, ${ }^{7}$ occurring in the neocortex as well as the brain stem. This may be an additional, underdiagnosed explanation for dementia in Parkinson's disease. ${ }^{8}$ Frontalsubcortical dysfunction may arise from deficits in the dopaminergic connections from the caudate nucleus and midbrain to the frontal cortex. ${ }^{9}$ There is no firm evidence that 